\title{
Estudio de los parámetros de proceso del anodizado por plasma químico en Ti-6Al-4V ELI para implantes dentales
}

\author{
Effect of parameters of micro-arc oxidation \\ process on Ti-6Al-4V ELI for dental implants
}

\author{
Anahí Azpeitia ${ }^{1}$, Adriana Lemos ${ }^{1}$, Kyung W. Kang ${ }^{1}$, \\ Carlos Llorente ${ }^{1,2}$, Pablo Bilmes ${ }^{1}$
}

\footnotetext{
${ }^{1}$ Laboratorio de Investigaciones de Metalurgia Física (LIMF), Facultad de Ingeniería, Universidad Nacional de La Plata, La Plata CP: 1900, Buenos Aires, Argentina.

${ }^{2}$ Comisión de Investigaciones Científicas de la Provincia de Buenos Aires - CICPBA, Buenos Aires, Buenos Aires. e-mail: azpeitiaanahi@gmail.com, adriana.kang@ing.unlp.edu.ar
}

\section{RESUMEN}

Con el objetivo de estudiar el efecto de la variación de algunos parámetros de proceso de anodizado por plasma químico (APQ) en muestras de Ti-6Al-4V para implantes dentales, se llevaron a cabo distintos tratamientos bajo diferentes condiciones de densidad de corriente, tiempo de exposición y composición química del electrolito; que produjeron recubrimientos superficiales bioactivos de óxido de titanio enriquecido en calcio y fósforo. Los tratamientos APQ se realizaron a partir de dos condiciones superficiales de partida: mecanizado y blastinizado. Luego de cada tratamiento APQ, se efectuó un tratamiento alcalino para evaluar su influencia sobre la bioactividad del recubrimiento obtenido. Para caracterizar las superficies se efectuaron análisis topográficos mediante microscopia electrónica de barrido, ensayos de adhesión a partir del test estándar Rockwell-C y evaluación de la bioactividad mediante ensayo de simulación en fluidos corporales (SBF). Los mejores resultados (recubrimientos uniformes y bioactivos con comportamiento satisfactorio en el ensayo de adhesión) fueron conseguidos con mayores tiempos de APQs en electrolitos de fosfóricosulfúrico y de fostato diácido de potasio. Asimismo, se evidenció que un blastinizado previo al APQ favorece la adhesión del recubrimiento; mientras que el tratamiento alcalino posterior al APQ mejora la bioactividad del mismo.

Palabras clave: Ti-6Al-4V, anodizado por plasma químico, implantes.

\section{ABSTRACT}

In order to study the variation effect of some parameters of micro-arc oxidation process (MAO) on Ti-6Al$4 \mathrm{~V}$ samples for dental implants, several treatments were carried out under different conditions of current density, exposure time and electrolyte chemical composition. These processes resulted in generated bioactive titanium oxide surface coatings enriched in calcium and phosphorus. The MAO treatment was perfomed from two starting surface conditions: machining and blasting. After each MAO treatment, an alkali treatment was performed to evaluate its influence on the bioactivity of the coating obtained. The surfaces were characterized by means of topographic analysis with scanning electron microscopy, coating adhesion from Rockwell-C standard test and bioactivity in a simulated body fluid test (SBF). The best results (uniform and bioactive coatings with satisfactory performance in the adhesion assay) were attained with longer treatment times and in phosphoric-sulfuric and potassium diacid phosphate electrolytes. Furthermore, the results showed that the blasting treatment previous to MAO process enhanced the coating adhesion while a subsequent alkaline treatment improved MAO bioactivity.

Keywords: Ti-6Al-4V, micro arc oxidation, implants. 


\section{INTRODUCCIÓN}

En la actualidad, gran parte del éxito clínico de los implantes se debe a mejoras en la gestión quirúrgica junto con los avances generados en materia de la comprensión de las interacciones biológicas. El éxito postimplantación se debe, principalmente, a la posibilidad de creación y mantenimiento de una interfase implante/hueso que permite la transferencia de carga y que se encuentra asociada al desarrollo de tejidos saludables [1].

Se ha demostrado que los sistemas biológicos tienen la capacidad de reconocer cualquier detalle a nivel molecular y debido a que las células no responden en forma directa a las condiciones superficiales sino que lo hacen a través de las moléculas adsorbidas en la superficie del implante, es importante conocer la influencia de la composición química, topografía y rugosidad superficial, energía superficial y propiedades fisicoquímicas superficiales de los implantes [2]. La energía superficial, mojabilidad (hidrofobicidad o hidrofilicidad), composición química, carga superficial y topografía, entre otros factores, tienen una fuerte influencia en la estabilidad a largo plazo del implante. Por otro lado, si bien la energía superficial de los biomateriales tiene una influencia en la adhesión y proliferación celular, en términos de orientación celular juegan un papel más relevante la textura, morfología y rugosidad de la superficie [3,4].

El titanio y sus aleaciones es el biomaterial de mayor uso como implante en odontología y traumatología. La extensa aplicación de este biomaterial se debe a que es uno de los metales más biocompatibles, superior a los aceros inoxidables y a las aleaciones base cobalto, con una excelente combinación de resistencia a la corrosión y alto desempeño mecánico. La resistencia a la corrosión del titanio se debe a su capacidad para formar una capa de óxido protectora, bioinerte, químicamente estable, continua y altamente adherente sobre su superficie [1]. No obstante, si bien el titanio es un material osteoconductor, su capacidad de inducir formación de hueso es bastante pobre o nula, a diferencia de los materiales bioactivos, como las apatitas, que en determinadas condiciones presentan características osteoinductoras, es decir, inducen o estimulan una diferenciación de células madre a células osteogénicas [5]. Es evidente que la respuesta de un biomaterial depende de sus propiedades superficiales y con el fin de mejorar su rendimiento en los sistemas biológicos, existe una urgente necesidad de desarrollar tratamientos y/o recubrimientos superficiales que incrementen la bioactividad de los implantes y, con ello, su rendimiento clínico.

Dentro de los numerosos tratamientos superficiales desarrollados, se encuentra el anodizado por plasma químico (APQ). Este tratamiento superficial permite generar recubrimientos de tipo cerámico en la superficie de diversos metales entre los cuales se encuentra el titanio. En el proceso de APQ, el ánodo, de material metálico, es inmerso en una solución acuosa, y se aplica una diferencia de potencial o corriente constante, permitiendo la ruptura dieléctrica. Utilizando la técnica de APQ, se obtienen recubrimientos con alta dureza, adhesión y resistencia al desgaste. Las propiedades físicas, químicas y mecánicas del recubrimiento están determinadas por los parámetros de proceso, como la composición y temperatura del electrolito, composición del metal, el voltaje, la densidad de corriente, el tiempo, etc [6]. De la misma manera, el tratamiento alcalino [1] ha despertado un interés creciente debido a su simplicidad y eficacia. La formación de un hidrogel de titanato de sodio amorfo sobre las superficies de titanio luego de los tratamientos alcalinos puede inducir la formación de apatita después de la inmersión durante un cierto tiempo en un fluido corporal simulado (SBF). En consecuencia, la combinación del proceso de APQ con el tratamiento alcalino produce superficies altamente bioactivas, con una prometedora perspectiva en la producción industrial debido a la simplicidad y bajo costo.

El objetivo del presente trabajo fue determinar los principales parámetros de proceso del anodizado por plasma químico (APQ) en Ti-6Al-4V, bajo diferentes condiciones de densidad de corriente, tiempo de exposición y composición química del electrolito, que produjeron recubrimientos superficiales de óxido de titanio enriquecido en calcio y fósforo. Para caracterizar las superficies se efectuaron observaciones topográficas mediante microscopía electrónica de barrido, ensayos de adhesión a partir del test estándar Rockwell-C [7] y evaluación de la bioactividad mediante ensayo de simulación en fluidos corporales (SBF) [8].

\section{MATERIALES Y MÉTODOS}

\subsection{Material de estudio}

Se utilizó como material de estudio la aleación Ti-6Al-4V ELI (Extra Low Interstitial) para aplicación quirúrgica (UNSR56401) ASTM F 136-02a. Se emplearon muestras cilíndricas de $8 \mathrm{~mm}$ de diámetro y $3 \mathrm{~mm}$ de espesor. Las condiciones superficiales de partida fueron: mecanizado con y sin blastinizado. Este último se realizó con partículas de fosfato de calcio con un tamaño promedio de $300 \mu \mathrm{m}$, durante dos minutos a 6 bar de 
presión.

\subsection{Procedimiento experimental}

Todas las muestras fueron previamente decapadas empleando una solución de ácido nítrico 0,2 M y ácido fluorhídrico $0,1 \mathrm{M}$ durante 1 minuto. Seguidamente se les aplicó el tratamiento de anodizado por plasma químico (APQ) en forma galvanostática, utilizando como referencia los parámetros de proceso (composición química del electrolito y la densidad de corriente) estudiados por WANXIA et al., QUINTERO et al. y GALVIS et al. [9-11]. Finalmente, luego de los tratamientos de APQ se realizaron dos enjuagues con agua bidestilada, secado al aire y envasado individual en films de polietileno. A continuación, se especifican los electrolitos utilizados y las condiciones en que se realizaron los tratamientos de APQ:

\section{A-. Fosfato de Calcio Anhidro $\left(\mathrm{CaHPO}_{4}\right) /$ Acetato de Calcio $\left(\mathrm{Ca}\left(\mathrm{CH}_{3} \mathrm{COO}\right)_{2}\right)$ \\ B-. Ácido Fosfórico/Ácido Sulfúrico: 0,25M de $\mathrm{H}_{2} \mathrm{SO}_{4}$ y 0,25M de $\mathrm{H}_{3} \mathrm{PO}_{4}$ \\ C-. Fosfato Biácido de Potasio: 0,15M Acetato de Calcio $\left(\mathrm{Ca}\left(\mathrm{CH}_{3} \mathrm{COO}\right)_{2}\right), 0,15 \mathrm{M}$ de Etilendiaminotetraacé- tico y $0,05 \mathrm{M}$ de Fosfato dihidrógeno de Potasio $\left(\mathrm{KH}_{2} \mathrm{PO}_{4}\right)$.}

En particular la selección de los electrolitos: "A" fosfato/acetato y "B" fosfórico/sulfúrico, se debió a que son los electrolitos utilizados para el anodizado por plasma químico (APQ) de Ti ASTM Grado 4 siendo importante analizar su aplicabilidad en la aleación Ti-6Al-4V.

En una primera instancia, se trabajó con el electrolito "C", bajo las siguientes condiciones: densidades de corriente de 25,50 y $75 \mathrm{~mA} / \mathrm{cm}^{2}$ y tiempos de 3 y 6 minutos. Bajo algunas de estas condiciones planteadas, se produjo un aumento localizado de la temperatura en la interfase electrolito/aire, provocando la interrupción del proceso, lo cual obligo a redefinir las condiciones experimentales, Tabla 1. Las siglas B (Blastinizado) y M (Mecanizado) indican las condiciones superficiales de partida.

Tabla 1: Parámetros de proceso de APQ utilizados en la primera etapa.

\begin{tabular}{c|c|c|c}
\hline ELECTROLITO & $\begin{array}{c}\text { SUPERFICIE } \\
\text { DE PARTIDA }\end{array}$ & $\begin{array}{c}\text { TIEMPO } \\
\text { (MINUTOS) }\end{array}$ & $\begin{array}{c}\text { DENSIDAD DE } \\
\text { CORRIENTE }\left(\mathbf{m A} / \mathbf{c m}^{2}\right)\end{array}$ \\
\hline “C” Fosfato biácido de potasio & $\mathrm{M}$ & 3 & 25 \\
\hline "C" Fosfato biácido de potasio & $\mathrm{M}$ & 6 & 25 \\
\hline "C" Fosfato biácido de potasio & $\mathrm{M}$ & 2,45 & 50 \\
\hline "C" Fosfato biácido de potasio & $\mathrm{M}$ & 2,5 & 75 \\
\hline "C" Fosfato biácido de potasio & $\mathrm{M}$ & 3 & 40 \\
\hline
\end{tabular}

A la luz de los resultados preliminares obtenidos en esta primera etapa, se fijó la densidad de corriente en 25 $\mathrm{mA} / \mathrm{cm}^{2}$ y el tiempo de proceso en: $1 ; 2 ; 2,5,3$ y 4 minutos, para los tres electrolitos en estudio, Tabla 2.

Tabla 2: Parámetros de proceso de APQ utilizados en la segunda etapa.

\begin{tabular}{c|c|c|c}
\hline ELECTROLITO & $\begin{array}{c}\text { SUPERFICIE } \\
\text { DE PARTIDA }\end{array}$ & $\begin{array}{c}\text { TIEMPO } \\
\text { (MINUTOS) }\end{array}$ & $\begin{array}{c}\text { DENSIDAD DE } \\
\text { CORRIENTE (mA/cm })\end{array}$ \\
\hline "A" Fosfato/acetato & M & 1 & 25 \\
\hline "A" Fosfato/acetato & M & 2 & 25 \\
\hline "A" Fosfato/acetato & M & 2,5 & 25 \\
\hline "A" Fosfato/acetato & M & 3 & 25 \\
\hline "A" Fosfato/acetato & B & 3 & 25 \\
\hline "A" Fosfato/acetato & M & 4 & 25 \\
\hline "A" Fosfato/acetato & B & 4 & 25 \\
\hline "B" Fosfórico/sulfúrico & M & 1 & 25 \\
\hline "B" Fosfórico/sulfúrico & M & 2 & 25 \\
\hline "B" Fosfórico/sulfúrico & M & 2,5 & 25 \\
\hline
\end{tabular}




\begin{tabular}{|c|c|c|c|}
\hline “B” Fosfórico/sulfúrico & M & 3 & 25 \\
\hline "B" Fosfórico/sulfúrico & $\mathrm{B}$ & 3 & 25 \\
\hline “B” Fosfórico/sulfúrico & M & 4 & 25 \\
\hline “B” Fosfórico/sulfúrico & $\mathrm{B}$ & 4 & 25 \\
\hline "C" Fosfato biácido de potasio & M & 1 & 25 \\
\hline "C" Fosfato biácido de potasio & M & 2 & 25 \\
\hline "C" Fosfato biácido de potasio & M & 2,5 & 25 \\
\hline "C" Fosfato biácido de potasio & M & 3 & 25 \\
\hline "C" Fosfato biácido de potasio & $\mathrm{B}$ & 3 & 25 \\
\hline "C" Fosfato biácido de potasio & M & 4 & 25 \\
\hline "C" Fosfato biácido de potasio & $\mathrm{B}$ & 4 & 25 \\
\hline
\end{tabular}

\subsection{Técnicas de caracterización}

Para la caracterización topográfica de las muestras se utilizó un microscopio electrónico de barrido ambiental FEI Quanta 200 (ESEM) y los microanálisis químicos se realizaron con un espectrómetro dispersivo en energías EDAX Apollo 40 (EDS) adosado al ESEM. La calidad y la resistencia adhesiva de los recubrimientos superficiales bioactivos de óxido de titanio enriquecido en calcio y fósforo se evaluaron mediante el ensayo de indentación Rockwell C, según la norma VDI 3198 [7]. Se realizaron dos indentaciones en cada muestra bajo estudio aplicando una carga de $150 \mathrm{Kg}$. Posteriormente, se realizaron las observaciones de las superficies mediante ESEM. La calidad de la adhesión del recubrimiento cerámico de las muestras en estudio se comparó con la clasificación suministrada por la norma mencionada, la cual define la calidad de adhesión en una escala que denomina HF1 a HF6. En general, HF1-HF4 define una adherencia suficiente del recubrimiento al sustrato, mientras que HF5 y HF6 representa una adherencia insuficiente.

Por otro lado, a un grupo de las muestras con APQ se les realizó un tratamiento alcalino en una solución $10 \mathrm{M}$ de $\mathrm{Na}(\mathrm{OH})$ durante $24 \mathrm{hs} \mathrm{a} 60^{\circ} \mathrm{C}$. Terminado el tratamiento alcalino, las muestras se enjuagaron con agua bidestilada y se secaron al aire.

Posteriormente, se evaluó la bioactividad mediante ensayo de simulación en fluidos corporales (SBF) durante 7 días de las muestras con APQ, con y sin tratamiento alcalino, que presentaron mejor comportamiento a la adhesión y homogeneidad superficial. El ensayo en SBF se llevó a cabo según la norma ISO 23317:2012 (E) [8]. Luego de los ensayos en SBF, se realizó la observación y análisis químico de las superficies resultantes mediante ESEM-EDS.

\section{RESULTADOS Y DISCUSIÓN}

\subsection{Anodizado por plasma químico (APQ)}

De los tratamientos realizados en la primera etapa sobre las muestras mecanizadas (sin blastinizado) (Tabla 1), los resultados obtenidos en el ensayo de adhesión, de acuerdo a la norma VDI 3198, muestran que los recubrimientos generados en fosfato biácido de potasio con densidades de corriente mayores a $40 \mathrm{~mA} / \mathrm{cm}^{2}$ y tiempo de ensayo de 3 minutos, como también los recubrimientos generados con densidades de corriente de $25 \mathrm{~mA} / \mathrm{cm}^{2}$ y 6 minutos de ensayo, no presentaron buena adherencia. La única condición que cumplió con los parámetros establecidos por la norma VDI 3198 fue la muestra realizada con densidad de corriente de 25 $\mathrm{mA} / \mathrm{cm}^{2}$ y un tiempo de 3 minutos. En Figura 1 se pueden observar los resultados obtenidos para esta última condición. La imagen izquierda corresponde a la topografía obtenida en APQ y la imagen derecha pertenece a una de las indentaciones obtenidas del ensayo de adhesión. 


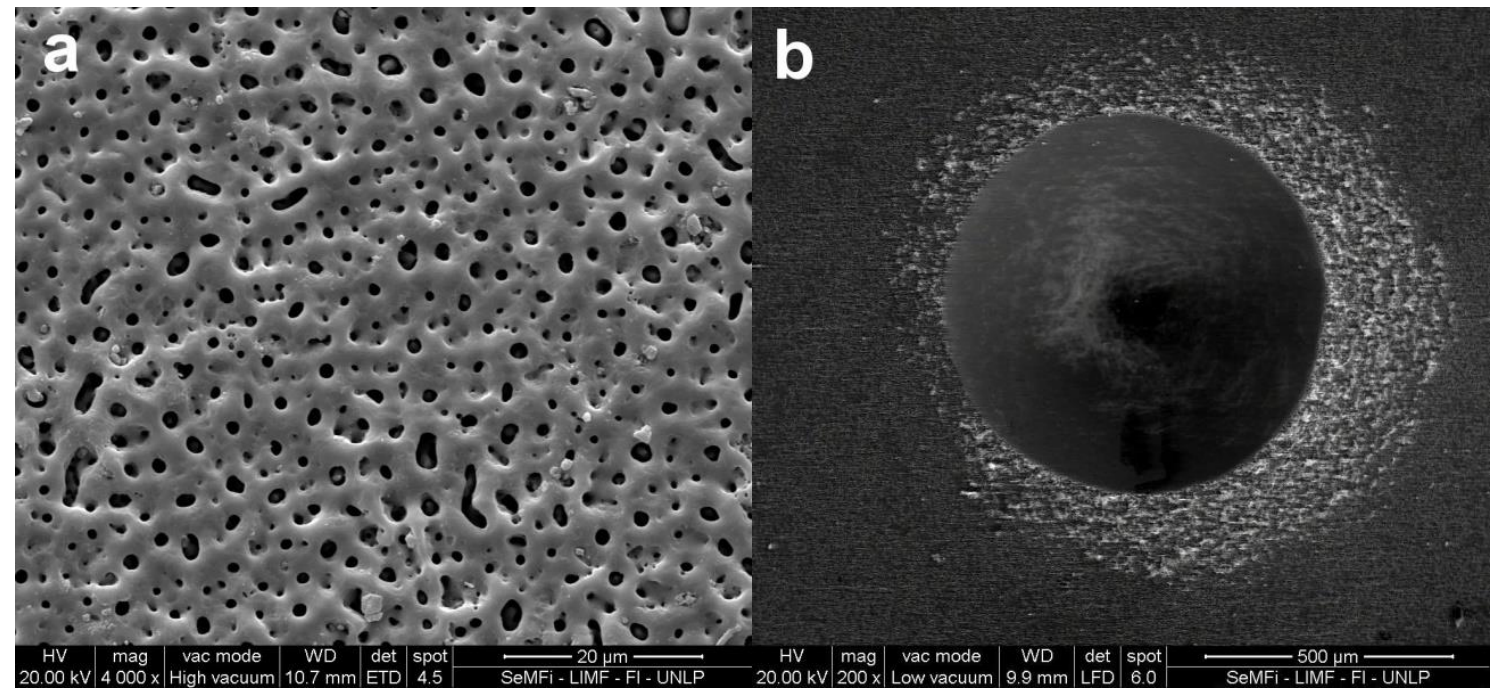

Figura 1: Imágenes de ESEM de la muestra con tratamiento APQ con electrolito " $C$ " fosfato biácido de potasio a $25 \mathrm{~mA} / \mathrm{cm}^{2}$ y 3 minutos: (a) Topografía (b) Indentación del ensayo de adhesión. El recubrimiento posee una adherencia suficiente según norma VDI 3198.

Asimismo, los resultados obtenidos en las muestras mecanizadas, ensayadas con los diferentes electrolitos, indican que el recubrimiento cerámico no es homogéneo con tiempos de ensayo inferior a 3 minutos (Tabla 2). Por otro lado, con densidad de corriente de $25 \mathrm{~mA} / \mathrm{cm}^{2}$ y 3 minutos de tratamiento se logran resultados satisfactorios. En la Figura 2 se pueden observar la topografía y las indentaciones del ensayo de adhesión de los recubrimientos obtenidos con los tres electrolitos.

De estos, el que presentó menor adhesión y homogeneidad de recubrimiento a $25 \mathrm{~mA} / \mathrm{cm}^{2}$ y 3 minutos de proceso, fue la muestra obtenida con el electrolito " $\mathrm{A}$ " fosfato/acetato.

Además, se pudo establecer que las muestras anodizadas en los electrolitos de "B" y " $\mathrm{C}$ ", con y sin blastinizado, a 4 minutos, presentaron un recubrimiento cerámico homogéneo y con el mejor comportamiento en adhesión. 


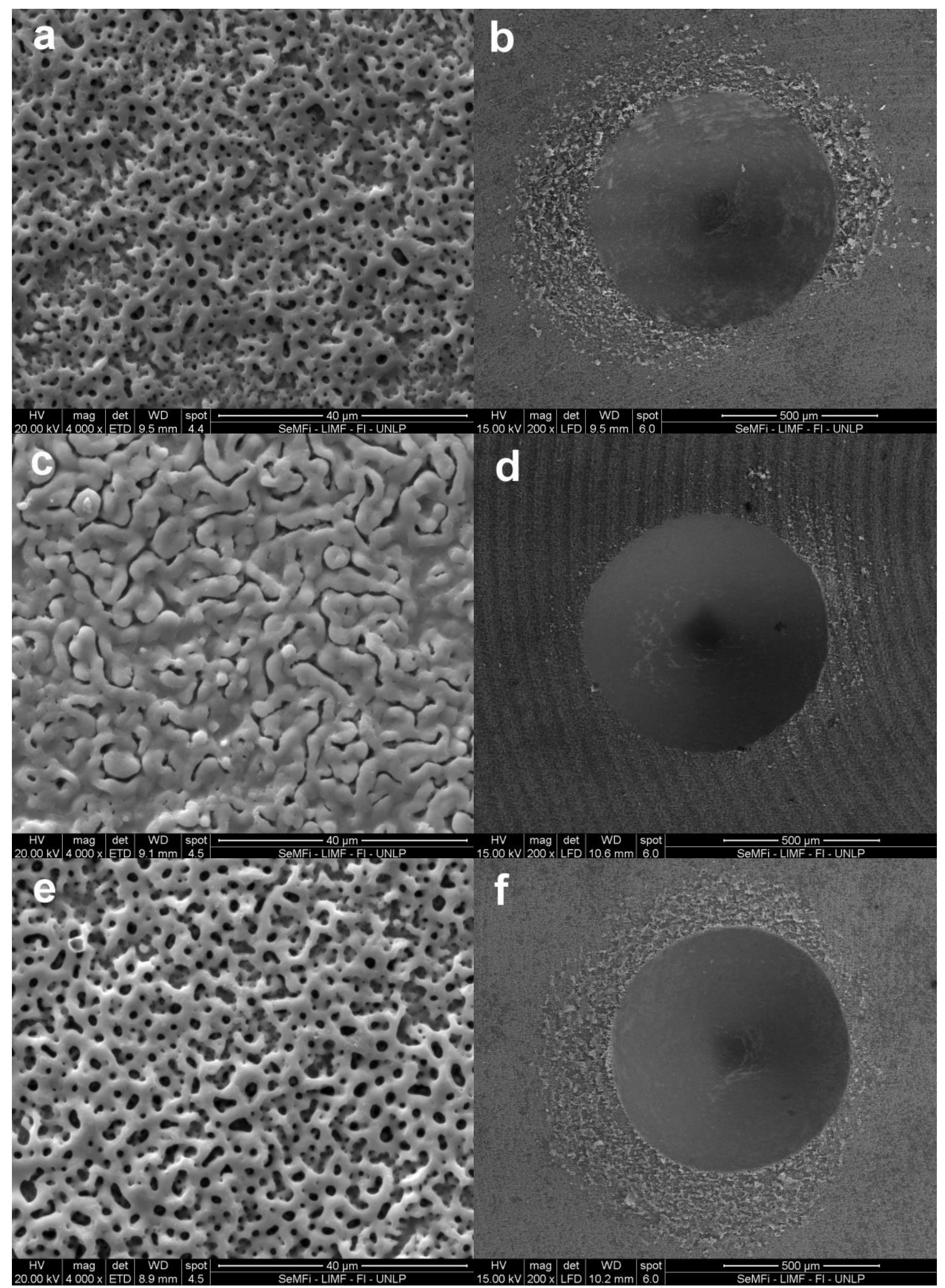

Figura 2: Imágenes de ESEM de las muestras con tratamiento APQ a $25 \mathrm{~mA} / \mathrm{cm}^{2}$ y 3 minutos. (a-b) Topografía e indentación del ensayo de adhesión del electrolito "A" fosfato/acetato (c-d) Topografía e indentación del ensayo de adhesión del electrolito "B" fosfórico/sulfúrico (e-f) Topografía e indentación del ensayo de adhesión del electrolito "C" fosfato biácido de potasio. La muestra con electrolito "A" presenta menor adhesión y homogeneidad de recubrimiento que los obtenidos con los electrolitos "B" $\mathrm{y}$ " $\mathrm{C}$ ". 
Por otro lado, las muestras mecanizadas (sin blastinizado) y las blastinizadas, ensayadas con el electrolito "A" a 3 y 4 minutos presentaron un recubrimiento inhomogéneo. En la Figura 3 se pueden observar las imágenes topográficas y de adhesión de las muestras con los tratamientos en los electrolitos de "B" fosfórico/sulfúrico y "C" fosfato biácido de potasio, con y sin blastinizado a 4 minutos. En todos los casos, las muestras cuyas superficies fueron previamente blastinizadas presentaron una mejor adherencia sustratorecubrimiento. Estos presentaron una topografía similar a las obtenidas por otros autores [9-13].

Si bien los recubrimientos obtenidos con el electrolito "A" fosfato/acetato fueron los que presentaron resultados poco satisfactorios en cuanto a adhesión, este no debe ser descartado siendo necesario realizar un trabajo experimental más amplio para establecer mejores condiciones operativas de densidad de corriente y tiempo para obtener un recubrimiento que cumpla con la adherencia y homogeneidad superficial.

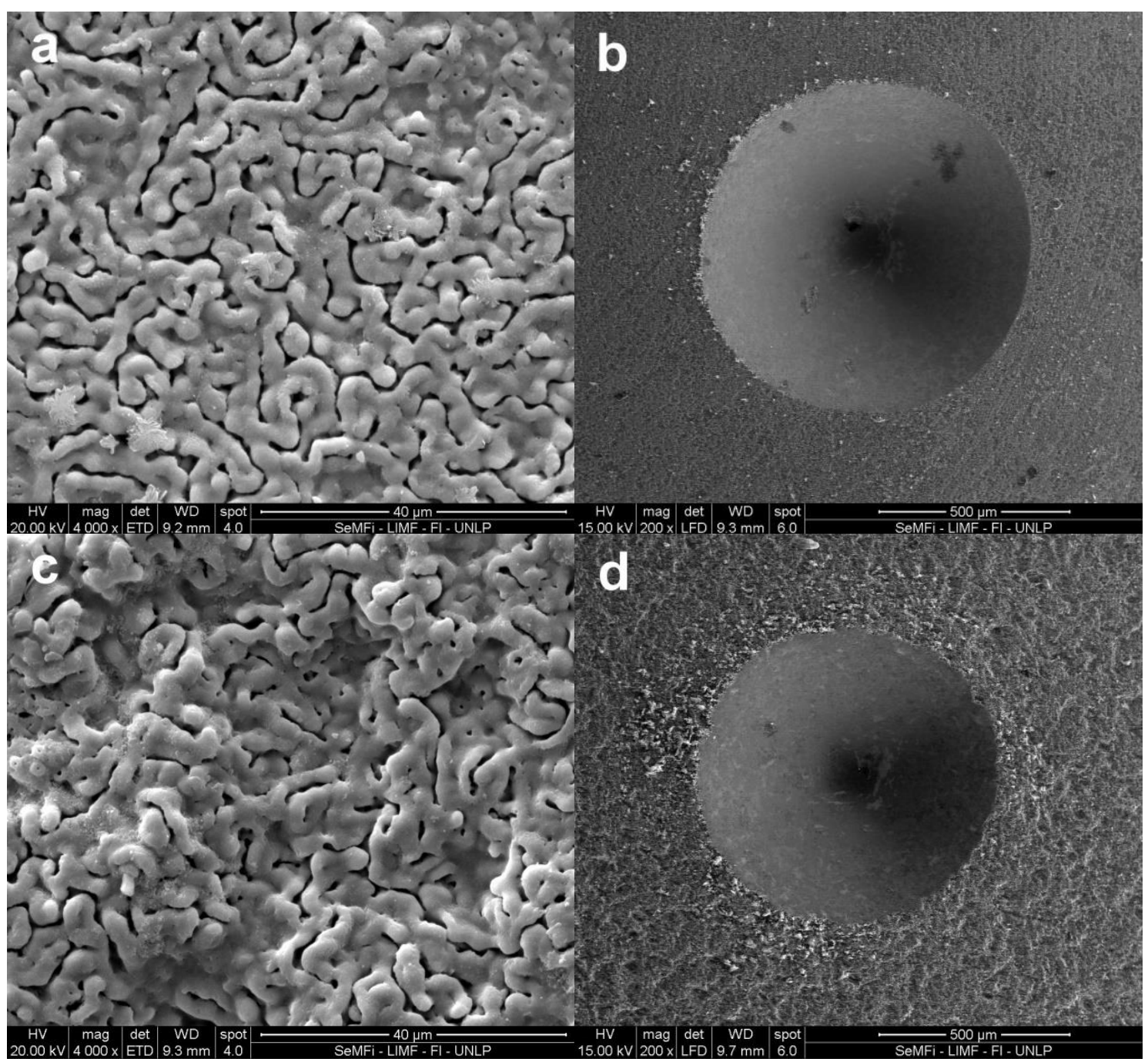




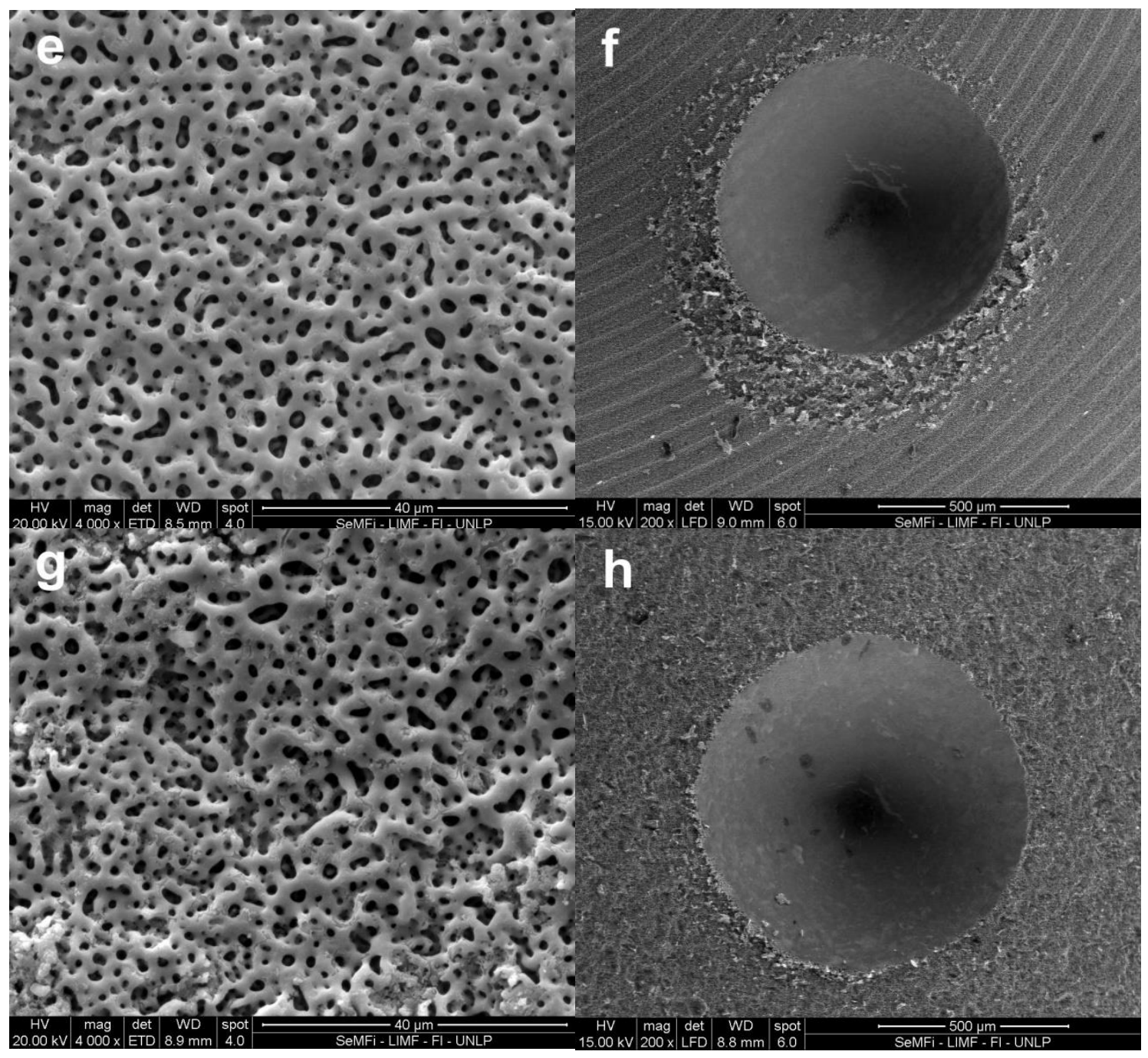

Figura 3: Imágenes de ESEM de las muestras con tratamiento APQ a $25 \mathrm{~mA} / \mathrm{cm}^{2}$ y 4 minutos. (a-b) Topografía e indentación del ensayo de adhesión del electrolito "B" fosfórico/sulfúrico sin blastinizado (c-d) Topografía e indentación del ensayo de adhesión del electrolito "B" fosfórico/sulfúrico con blastinizado (e-f) Topografía e indentación del ensayo de adhesión del electrolito "C" fosfato biácido de potasio sin blastinizado (g-h) Topografía e indentación del ensayo de adhesión del electrolito "C" fosfato biácido de potasio con blastinizado. Las muestras cuyas superficies fueron previamente blastinizadas presentaron una mejor adherencia sustrato-recubrimiento.

\subsection{Tratamiento alcalino y SBF}

La simulación en fluidos corporales (SBF), según la norma ISO 23317:2012 (E), permite estudiar in vitro la formación de apatita en la superficie de las probetas y predecir la bioactividad de las mismas. Se evaluó la bioactividad en SBF de las muestras obtenidas con los electrolitos "B" y "C", con y sin blastinizado, a 4 minutos. A un grupo de dichas muestras se les realizó un tratamiento alcalino previo al ensayo en SBF. Algunos autores [13-15] demuestran que funcionalizar las superficies con un tratamiento alcalino antes del ensayo en SBF ayuda a la precipitación de apatita en menores tiempos de exposición.

La respuesta bioactiva del recubrimiento, observada a través del ensayo de SBF, está asociada a la funcionalización de la superficie con los grupos Ti-OH, que se producen como consecuencia del tratamiento alcalino en $\mathrm{Na}(\mathrm{OH})[15]$.

En la Figura 4 se pueden observar las superficies luego de llevar a cabo el tratamiento alcalino en $\mathrm{Na}(\mathrm{OH})$. La formación de grietas en la superficie se debe a la deshidratación del hidrogel formado [13]. La superficie tiene una topografía similar a la obtenida por ZHAO et al. [13]. 


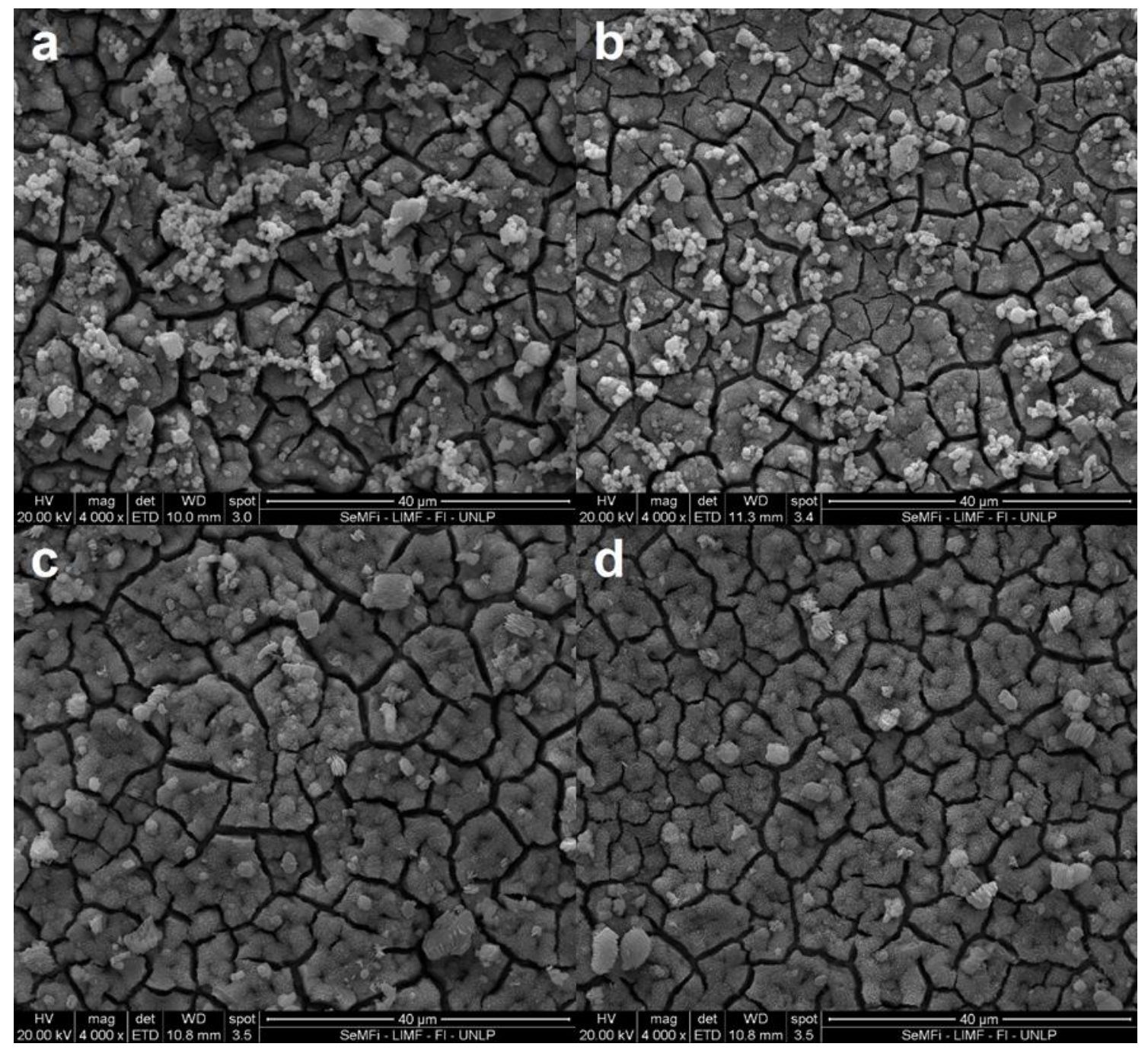

Figura 4: Imágenes de ESEM de las muestras con tratamiento APQ a $25 \mathrm{~mA} / \mathrm{cm}^{2}$ y 4 minutos y posterior tratamiento alcalino en $\mathrm{Na}(\mathrm{OH})$. (a) "B" fosfórico/sulfúrico con blastinizado (b) "B" fosfórico/sulfúrico sin blastinizado (c) "C" fosfato biácido de potasio con blastinizado (d) " $\mathrm{C}$ " fosfato biácido de potasio sin blastinizado. Las grietas que se pueden observar en la superficie se deben a la deshidratación del hidrogel formado.

En la Figura 5 se puede observar que las muestras con tratamiento alcalino presentan un precipitado bioactivo homogéneo en toda la superficie después de 7 días de inmersión en SBF. En las muestras sólo con anodizado por plasma químico (APQ) no se observó este precipitado bioactivo en las superficies. 


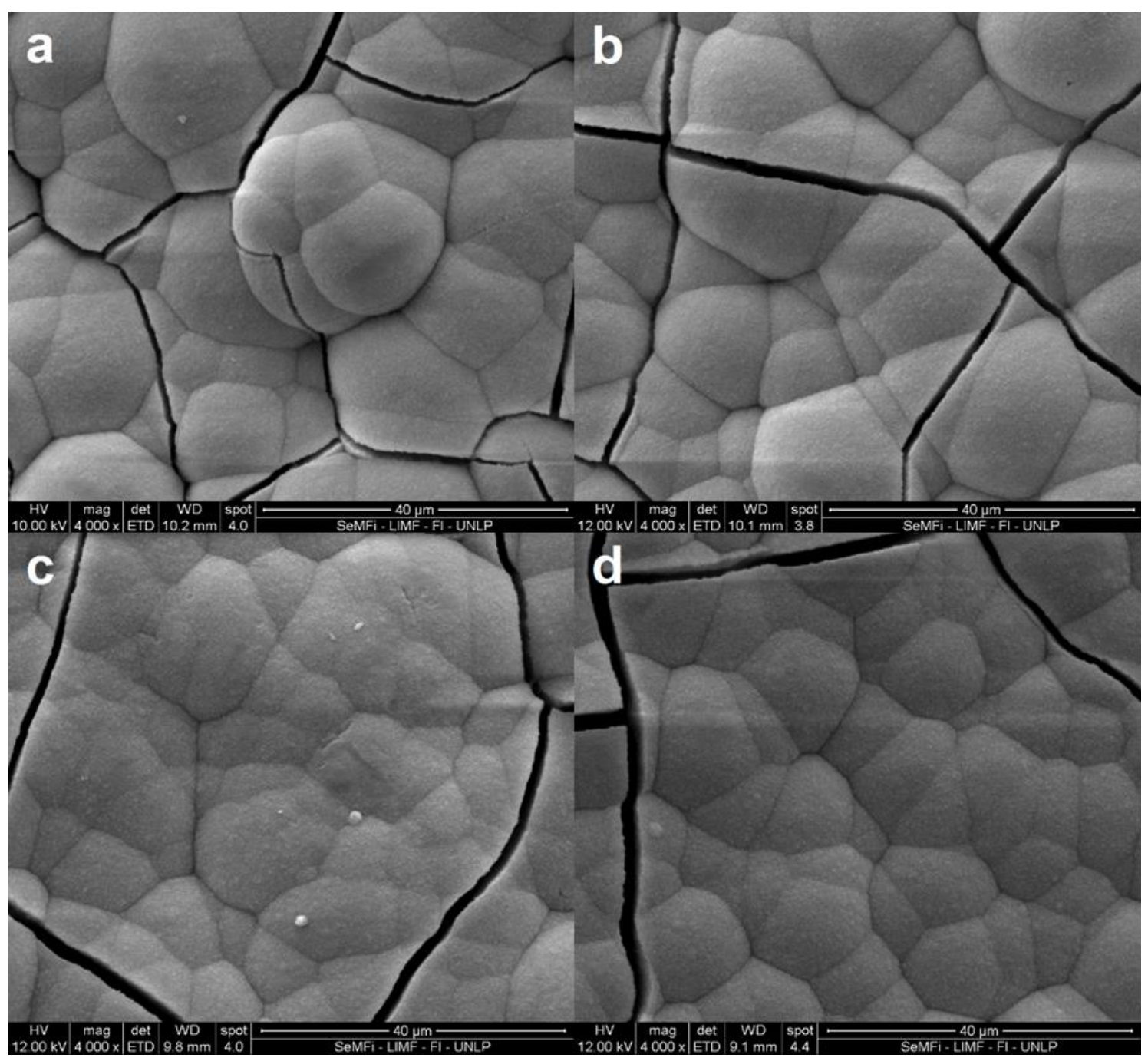

Figura 5: Imágenes de ESEM de las superficies obtenidas luego del ensayo de SBF de las muestras con tratamiento APQ a $25 \mathrm{~mA} / \mathrm{cm}^{2}$ y 4 minutos y posterior tratamiento alcalino en $\mathrm{Na}(\mathrm{OH})$. (a) "B" fosfórico/sulfúrico con blastinizado (b) "B" fosfórico/sulfúrico sin blastinizado (c) "C" fosfato biácido de potasio con blastinizado (d) "C" fosfato biácido de potasio sin blastinizado. Se puede apreciar un precipitado bioactivo homogéneo en las superficies después de 7 días de inmersión en SBF.

En la tabla 3 se muestran los resultados obtenidos del análisis realizado con EDS en las superficies presentadas en la figura 5. Si bien las relaciones atómicas de $\mathrm{Ca} / \mathrm{P}$ de las capas de apatita depositadas son menores que las de la apatita estequiométrica (1.67), este valor se encuentra dentro del error de la técnica EDS.

Tabla 3: Análisis EDS de la capa de hidroxiapatita formada luego del ensayo de SBF a 7 días de inmersión en las muestras con tratamiento APQ a $25 \mathrm{~mA} / \mathrm{cm}^{2}, 4$ minutos y posterior tratamiento alcalino en $\mathrm{Na}(\mathrm{OH})$.

\begin{tabular}{c|c|c|c}
\hline \multirow{2}{*}{ TRATAMIENTO } & \multicolumn{2}{|c|}{ ELEMENTOS AT\% } & \multirow{2}{*}{ RELACIÓN CA/P } \\
\cline { 2 - 3 } & Ca & P & \\
\hline Fosfórico/sulfúrico, CB & 24,81 & 16,03 & 1.6 \\
\hline Fosfórico/sulfúrico, SB & 21,90 & 14,59 & 1.5 \\
\hline Fosfato biácido de potasio, CB & 24,40 & 16,44 & 1.5 \\
\hline Fosfato biácido de potasio, SB & 23,95 & 16,01 & 1.5 \\
\hline
\end{tabular}




\section{CONCLUSIONES}

A partir de los análisis realizados mediante ESEM de todas las muestras, en sus diferentes condiciones, se evidencia que las superficies que presentan un recubrimiento cerámico uniforme corresponden a las muestras tratadas con los electrolitos de fosfórico/sulfúrico y fosfato biácido de potasio, mientras que el electrolito de fosfato/acetato da como resultado un recubrimiento heterogéneo.

Según lo dispuesto en la norma VDI 3198, los parámetros de APQ dados por densidad de corriente de $25 \mathrm{~mA} / \mathrm{cm}^{2}$ y 4 minutos de tratamiento, determinan un comportamiento favorable del recubrimiento obtenido frente al ensayo de adhesión con el metal base. Por otro lado, la incorporación de un tratamiento de blastinizado previo, mejora la respuesta en adhesión.

Por último, el ensayo en SBF a 7 días, demostró que el tratamiento alcalino en $\mathrm{Na}(\mathrm{OH})$ posterior al APQ mejora la bioactividad formando una capa de apatita homogénea en las superficies.

\section{AGRADECIMIENTOS}

Los autores quieren expresar su agradecimiento a la empresa Kinetical SRL por el suministro de los materiales utilizados en este trabajo y al Servicio de Microscopía Electrónica de Barrido y Microanálisis (SEMFi) del LIMF.

\section{BIBLIOGRAFÍA}

[1] KULKARNI, M., MAZARE, A., SCHMUKI, P., IGLIČ, A. "Biomaterial surface modification of titanium and titanium alloys for medical applications", In: Seifalian, A., de Mel, A., Kalaskar, D.M. (eds), Nanomedicine, led., Chapter 5, Manchester, United Kingdom, One Central Press, 2014.

[2] GORIAINOV, V., COOK, R., LATHAM, J.M., et al., "Bone and metal: An orthopaedic perspective on osseointegration of metals", Acta Biomaterialia, v. 10, n. 10, pp. 4043-4057, Jun. 2014.

[3] ALBREKTSSON, T., WENNERBERG, A., "Oral implant surfaces: Part I - Review focusing on topographic and chemical properties of different surfaces and in vivo responses to them", The International Journal of Prosthodontics, v. 17, n. 5, pp. 536-543. Sep-Oct. 2004.

[4] ALBREKTSSON, T., WENNERBERG, A., "Oral implant surfaces: Part II - Review focusing on clinical knowledge of different surfaces", The International Journal of Prosthodontics, v. 17, n. 5, pp. 544-564. SepOct. 2004.

[5] VALLET REGI, M., MUNUERA, L., Biomateriales aquí y ahora, 1 ed., Madrid, Dykinson, 2000.

[6] WANG, Y., YU, H., CHEN, C., et al., "Review of the biocompatibility of micro-arc oxidation coated titanium alloys", Materials and design, v. 85, pp. 640-652, Nov. 2015.

[7] VEREIN Deutscher Ingenieure Normen, VDI 3198, VDI-Verlag, Dusseldorf, 1991.

[8] IMPLANTS for surgery - In vitro evaluation for apatite-forming ability of implant materials, ISO 23317:2012.

[9] WANXIA, T., JIKANG, Y., GANG, Y., et al., "Effect of Electrolytic Solution Concentrations on Surface Hydrophilicity of Micro-Arc Oxidation Ceramic Film Based on Ti6Al4V Titanium Alloy", Rare Metal Materials and Engineering, v. 43, n. 12, pp. 2883-2888, Dic. 2014.

[10] QUINTERO, D., GALVIS, O., CALDERÓN, J.A., et al., "Effect of electrochemical parameters on the formation of anodic films on commercially pure titanium by plasma electrolytic oxidation", Surface \& Coatings Technology, v. 258, pp. 1223-1231, Nov. 2014.

[11] GALVIS, O.A., QUINTERO, D., CASTAÑO, J.G., et al., "Formation of grooved and porous coatings on titanium by plasma electrolytic oxidation in $\mathrm{H}_{2} \mathrm{SO}_{4} / \mathrm{H}_{3} \mathrm{PO}_{4}$ electrolytes and effects of coating morphology on adhesive bonding", Surface \& Coatings Technology, v. 269, pp. 238-249, May. 2015.

[12] SHI, X., WANG, Q., WANG, F., et al., "Effects of electrolytic concentration on properties of micro-arc film on Ti6Al4V alloy", Mining Science and Technology, v. 19, n. 2, pp. 220-224, Mar. 2009.

[13] ZHAO, G., XIA, L., ZHONG, B., et al., "Effect of alkali treatments on apatite formation of microarcoxidized coating on titanium alloy surface", Transactions of Nonferrous Metals Society of China, v. 25, n. 4, pp. 1151-1157, Apr. 2015.

[14] LI, N., XIAO, G., LIU, B., et al., "Rapid deposition of spherical apatite on alkali-heat treated titanium in modified simulated body fluid at high temperature", Surface \& Coatings Technology, v. 301, pp. 121-125, Sep. 2016. 
[15] LI, J., LIU, X. "Chemical surface modification of metallic biomaterials", In: Wen, C. (ed), Surface Coating and Modification of Metallic Biomaterials, 1 ed., chapter 5, Amsterdam, Netherlands, Elsevier, 2015. 